\title{
OCT Angiography of the Choriocapillaris in Central Serous Chorioretinopathy: A Quantitative Subgroup Analysis
}

\author{
Bertan Cakir · Michael Reich · Stefan Lang - Anima Bühler • \\ Christoph Ehlken · Bastian Grundel • Milena Stech • Sabine Reichl • \\ Andreas Stahl · Daniel Böhringer · Hansjürgen Agostini · \\ Clemens Lange
}

Received: October 1, 2018 / Published online: January 7, 2019

(C) The Author(s) 2019

\section{ABSTRACT}

Introduction: To quantify optical coherence tomography angiography (OCTA) signal changes at the level of the choriocapillaris (CC) in patients with different stages of central serous chorioretinopathy (CSC) and to explore any correlation between subretinal fluid (SRF) and retinal pigment epithelium (RPE) alterations and the OCTA CC signal.

Methods: One hundred one CSC eyes and 42 healthy control eyes were included in this retrospective study. CSC patients were allocated into four groups: acute, non-resolving, chronic atrophic and inactive CSC. CC OCTA images (AngioPlex $^{\circledR}$, Zeiss) were automatically quantified using an image-processing algorithm. Spatial correlation analysis of OCTA signals was performed by overlapping macular edema

Enhanced digital features To view enhanced digital features for this article go to https://doi.org/10.6084/ m9.figshare.7467146.

Electronic supplementary material The online version of this article (https://doi.org/10.1007/s40123018-0159-1) contains supplementary material, which is available to authorized users.

B. Cakir · M. Reich · S. Lang · A. Bühler · C. Ehlken

· B. Grundel · M. Stech · S. Reichl - A. Stahl .

D. Böhringer · H. Agostini · C. Lange ( $\varangle)$

Eye Center, Medical Center, Faculty of Medicine,

University of Freiburg, Freiburg, Germany

e-mail: clemens.lange@uniklinik-freiburg.de heatmaps and fundus autofluorescence images with corresponding OCTA images.

Results: Active CSC subgroups demonstrated significantly more increased and decreased flow pixels in the CC compared with controls $(p<0.0001)$. No significant OCTA changes were seen within the active CSC groups or between the inactive and healthy subgroup. Spatial correlation analysis revealed a decreased OCTA signal in the SRF area and an increased signal outside the SRF area in acute CSC. Areas of RPE atrophy co-localized with areas of increased choriocapillaris OCTA signal, while areas with RPE alterations exhibited a normal signal compared with unaffected RPE.

Conclusion: The decreased OCTA signal in the area of SRF in acute CSC could be evidence of localized CC hypoperfusion or due to shadowing artifacts. The missing CC OCTA changes in altered RPE adjacent to atrophy argues against CC injury. Studies with higher resolution and optimized image acquisition are warranted to further validate our findings.

Keywords: Central serous chorioretinopathy; Choriocapillaris; OCT angiography

\section{INTRODUCTION}

Central serous chorioretinopathy (CSC) ranks after age-dependent macular degeneration, diabetic retinopathy, and retinal vein occlusion as 
one of the most common vision-threatening retinopathies, with an annual incidence of $1: 10,000[1,2]$. CSC is characterized by localized subretinal fluid (SRF) at the posterior pole that typically resolves spontaneously within a few months [3]. In chronic cases, however, SRF can persist, damaging the photoreceptors and retinal pigment epithelium (RPE) and resulting in irreversible vision loss. Based on the onset of symptoms and the retinal and RPE changes that result, CSC can be subgrouped into several forms: an acute form with self-resolving SRF, a non-resolving form with SRF persisting longer than 4 months, a chronic atrophic form revealing widespread RPE atrophy with or without fluid, and an inactive form without SRF [4].

The pathogenesis of CSC is still poorly understood; however, most existing investigations suggest that the primary pathology occurs within the choroid and RPE-choriocapillaris (CC) complex. Animal and clinical studies demonstrate that overstimulation of the mineralocorticoid pathway in choroidal vessels leads to choroidal vasodilatation, RPE disruption, and fluid leakage under the retina $[5,6]$. The typical diagnostic clinical workup of CSC consists of a basic slit-lamp examination, optical coherence tomography (SD-OCT), fundus autofluorescence (FAF), fluorescence angiography (FAG) and indocyanine green angiography (ICG). However, none of these methods provide a detailed, high-resolution image of the CC to enable insight into the currently proposed pathomechanism.

OCT angiography (OCTA) is a new non-invasive imaging technique which uses the motion contrast of moving blood components instead of intravenous dye to create images of the retinal and choroidal vasculature. Compared with dye-based angiography, OCTA is unable to detect plasma movements and vascular leakage. The advantages of OCTA include its non-invasiveness, short examination time, and capability to generate high-resolution volumetric angiography scans of the retina and choroid [7-13].

Since its introduction, several studies have used OCTA to investigate the CC in small cohorts of patients with CSC and reported changes in the form of decreased and/or increased flow [8, 12, 14-17]. None of these studies, however, have provided quantitative data or investigated the CC signal in different disease stages. Furthermore, none have examined the interdependence between SRF and RPE changes and the OCTA signal in patients with CSC. Thus, in this retrospective study, we sought to quantify OCTA signal changes at the level of the CC in patients presenting with different stages of CSC and to explore any correlation between SRF and RPE alterations and the OCTA signal.

\section{METHODS}

\section{Subjects}

The charts of all CSC patients who underwent OCT angiography as part of their standard imaging protocol between December 2015 and April 2017 during their visit at the Eye Center of the University of Freiburg were reviewed retrospectively. Patients with concomitant maculopathies such as age-related macular degeneration, diabetic retinopathy, or other retinopathies were excluded from this study. Patient characteristics such as gender, date of birth, pre-existing conditions, initial diagnosis, previous treatment, duration of symptoms, and OCT findings such as intraretinal fluid, intraretinal deposits, and pigment epithelium deviation were documented. In addition to the basic ophthalmic examination, a fundus autofluorescence image (FAF) was taken of all patients. Secondary choroidal neovascularization was excluded in chronic CSC cases with additional FAG and ICG. Based on Daruich and colleagues' classification scheme [4], patients were classified according to their pigment epithelium status and the duration of their symptoms into in one of the four following groups: (1) acute CSC with SRF lasting < 4 months; (2) non-resolving CSC with SRF persisting > 4 months; (3) chronic atrophic CSC with extensive pigment epithelium atrophy with or without SRF, and (4) inactive CSC without SRF but presenting signs of a previous CSC episode (Table 1). Age-matched healthy 
Table 1 CSC classification according to Daruich et al. [4]

\begin{tabular}{llll}
\hline CSC stage & SRF & $\begin{array}{l}\text { SRF duration of } \\
\text { current episode }\end{array}$ & RPE status \\
\hline Acute & + & $<4$ month & Normal RPE or RPE alterations without atrophy \\
Non-resolving & + & $>4$ month & Normal RPE or RPE alterations without atrophy \\
Atrophic & + or - & NA & Widespread RPE atrophy with or without gravitational tracks \\
Inactive & - & NA & $\begin{array}{l}\text { Signs of previous CSC episode with RPE alterations without } \\
\text { atrophy }\end{array}$ \\
\hline
\end{tabular}

control subjects with visual acuity $>0.8$ were included as controls. All procedures performed in studies involving human participants were in accordance with the Freiburg University Hospital institutional review board and with the 1964 Helsinki Declaration and its later amendments or comparable ethical standards. Informed consent was obtained from all individual participants included in the study.

\section{Imaging and Image Analysis}

OCTA images were obtained with a commercially available OCTA system (Zeiss AngioPlexTM $\left.^{\circledR}\right)$. This device has an A-scan rate of 68,000 A-scans per second and uses the Optical Microangiography $\left(\mathrm{OMAG}^{\circledR}\right)$ algorithm for decorrelation signal detection. An advantage over other current OCTA algorithms is that it relies on both amplitude and phase in the OCTA signals to extract the blood flow information. A real-time image stabilizer (FastTracTM) ensures minimization of movement artifacts. Furthermore, a built-in software was used to eliminate positive artifacts created from the superficial vascular layers. Each patient received a $6 \times 6 \mathrm{~mm}^{2}$ volume scan. For OCTA visualization of the CC layer, a $30-\mu \mathrm{m}$ parallel slab between 30 and $60 \mu \mathrm{m}$ below the inner RPE was manually selected for each patient. In cases of pigment epithelium detachments (PED), the slab did not follow the PED.

Abnormal CC decorrelation signals were quantified using a self-designed image processing algorithm programmed in $\mathrm{R}$ language (www.r-project.org) as described previously
[17]. Briefly, images were processed via a Gaussian blur and morphologic hdome operator, and each image's average gray-scale value was measured. Pixels with increased or decreased gray-scale value were detected and color-coded in red or green, respectively. The threshold for the color coding was calculated for each OCTA image by using the averaged grayscale value of all pixels (brightness of pixel) plus or minus a constant and pre-defined threshold value. The pre-defined threshold value was visually determined using numerous exemplary OCT-A images as previously described [17]. Before quantifying the pixel counts, shadowing artifacts of the inner retinal vessels were manually removed from each image. The counts of red and green pixel were used for statistical analyses.

To analyze the spatial correlation between SRF and the OCTA CC signal in patients with acute CSC, the color-coded CC images were aligned with the corresponding heat map image containing macular thickness information obtained from the Zeiss AngioplexTM ${ }^{\circledR}$. The area with macular thickness $>450 \mu \mathrm{m}$ was defined as the region of interest to ensure spatial correlation analysis only in areas with significant SRF. This area was then automatically outlined by the program. Red and green pixel counts within and outside the area were recorded and used for statistical analyses. Similarly, areas revealing RPE changes in patients with chronic atrophic CSC were determined on FAF images and aligned with corresponding CC flow images using the superficial vascular plexus for both modalities as landmarks. Two areas 
showing RPE changes were manually outlined: (1) areas with obvious dark-appearing RPE atrophy; (2) areas with increased FAF signal surrounding the RPE atrophy. Pixel counts of the automated color-coded images within and outside the regions were used for statistical analysis.

\section{Statistical Methods}

Statistical analysis was performed using GraphPad Prism 6 (GraphPad Software, Inc., La Jolla, CA, USA). Distribution of our data set was analyzed by applying the D'Agostino-Pearson's omnibus and Kolmogorov-Smirnov normality test. We opted for the Mann-Whitney $U$ test for comparison between two groups since the data were not normally distributed. Statistical analysis for multiple groups was performed using the non-parametric ANOVA/Bonferroni multiple comparison test. Data were considered significant at $p<0.05$.

\section{RESULTS}

\section{Patient Characteristics}

A total of 101 eyes from 78 CSC patients and 42 eyes from 34 controls were included in this study. Average age of the CSC population was 51 years (range $30-80$ ) compared with 49 years (range 24-79) in the control group. For subgroup analyses, CSC patients were allocated into the aforementioned four groups. Only patients with indisputable characteristics were allocated in the subgroups. Patients whose symptoms were of uncertain duration were excluded from the subgroup analysis. Thirtyseven eyes were classified as acute CSC, 37 as non-resolving CSC, 11 as atrophic CSC, and 16 as inactive CSC. For a detailed characterization of all patients, see Table 2 .

\section{OCTA Analysis of the Choriocapillaris}

Patients with CSC demonstrated both areas with increased and decreased OCTA signal at the level of the CC. The number of pixels with increased
OCTA signal was significantly higher in patients with CSC (mean $43,238 \pm$ SD 19,568) than in control subjects $(23,348 \pm 12,226 ; p<0.0001)$. Similarly, the number of pixels with decreased OCTA signal was more pronounced in CSC $(30,243 \pm 15,793)$ than in control patients $(12,970 \pm 7454 ; p<0.0001)$. Subgroup analysis revealed increased and decreased OCTA signal at the level of the CC in acute, non-resolving, and atrophic CSC patients compared with the inactive form of CSC and healthy controls. The number of pixels with increased OCTA signal was similar in patients with acute CSC $(44,214 \pm$ $20,020)$, non-resolving CSC $(46,903 \pm 21,136)$, and chronic atrophic CSC $(43,641 \pm 12,234)$ and higher compared with patients with inactive CSC $(30,566 \pm 11,403)$ and healthy controls $(23,348 \pm 12,226$, ANOVA $p<0.0001)$. The number of pixels with decreased OCTA signal was comparable in patients with acute CSC $(31,991 \pm 18,020)$, non-resolving CSC $(30,564 \pm$ $13,758)$, and chronic atrophic CSC $(34,578 \pm$ 9352) and significantly higher than in healthy controls $(12,970 \pm 7454$; ANOVA $p<0.0001$; Fig. 1).

\section{Spatial Correlation Between Subretinal Fluid and Flow Signal in Patients with Acute CSC}

To explore the spatial correlation between SRF and OCTA signal, we included a total of 15 patients who had classic acute CSC defined by normal to minor RPE changes, a dome-shaped accumulation of central subretinal fluid, and symptoms lasting $<4$ months. Patients with acute CSC demonstrated an inhomogeneous and weaker OCTA signal in the SRF area and a stronger OCTA signal outside the SRF area (Fig. 2a-d). Within the SRF area, the percentage of pixels with decreased OCTA signal was significantly higher than that of pixels with increased OCTA signal $(10.2 \% \pm 5.7$ vs. $2.7 \% \pm 1.0 ; p<0.001)$. In the surrounding area without SRF, we detected the opposite, namely a significantly higher percentage of pixels with increased OCTA signal compared with decreased signal $(7 \% \pm 3.7$ vs. $1.8 \% \pm 1.0$; $p<0.0001$; Fig. 2e). The mean ratio of increased 
Table 2 Patient characteristics

\begin{tabular}{lllllll}
\hline & $\begin{array}{l}\text { All CSC patients } \\
(\boldsymbol{n}=101)\end{array}$ & $\begin{array}{l}\text { Acute CSC } \\
(\boldsymbol{n}=\mathbf{3 7})\end{array}$ & $\begin{array}{l}\text { Non-resolving } \\
\mathbf{C S C}(\boldsymbol{n}=\mathbf{3 7})\end{array}$ & $\begin{array}{l}\text { Atrophic CSC } \\
(\boldsymbol{n}=\mathbf{1 1})\end{array}$ & $\begin{array}{l}\text { Inactive CSC } \\
(\boldsymbol{n}=\mathbf{1 6})\end{array}$ & $\begin{array}{l}\text { Healthy } \\
\text { controls } \\
(\boldsymbol{n}=\mathbf{4 2})\end{array}$ \\
\hline Age $(\bar{x}$, range $)$ & $51(30-80)$ & $48(35-80)$ & $51(30-70)$ & $60(57-62)$ & $50(33-70)$ & $48(24-79)$ \\
Gender $(\mathrm{M} / \mathrm{F})$ & $83 / 18$ & $34 / 3$ & $28 / 9$ & $10 / 1$ & $11 / 5$ & $14 / 28$ \\
VA $(\bar{x})$ & 0.71 & 0.75 & 0.71 & 0.26 & 0.97 & 0.99 \\
\hline
\end{tabular}

to decreased OCTA signal was $-0.5 \pm 0.3$ in the area with SRF and $0.6 \pm 0.3$ outside the area of SRF $(p<0.0001)$. Taken together, these data indicate that increased OCTA signals dominate the area without SRF, while decreased OCTA signals are predominantly found in the area with SRF in patients with acute CSC (Fig. 2f).

\section{Spatial Correlation Between RPE Atrophy and Flow Signal in Patients with Atrophic CSC}

To determine the interdependence between RPE atrophy and the OCTA signal in the CC, we included a total of six patients with chronic atrophic CSC defined by widespread RPE and photoreceptor atrophy without subretinal fluid. We found that areas of RPE atrophy co-localized with areas of increased CC OCTA signal, while areas revealing RPE alterations defined by higher autofluorescence exhibited a normal OCTA signal compared with unaffected RPE in the same eye (Fig. 3a-d). Within the area of RPE atrophy, the percentage of pixels sending an increased OCTA signal was significantly higher than that of the unaffected RPE area in the corresponding eye $(11.7 \% \pm 4.9 \% \quad$ vs. $2.5 \pm 1.4 \% ; p<0.001)$. We found no significant difference in the number of pixels with decreased OCTA signal among areas of RPE atrophy, RPE alteration, and unaffected RPE (Fig. 3e). The mean ratio of increased to decreased OCTA signals was $0.21 \pm 0.26$ in the area of RPE atrophy, $0.15 \pm 0.19$ in the area of RPE alteration, and $-0.03 \pm 0.2$ in the area of normal RPE, which underlines our finding that RPE atrophy is associated with increased OCTA signal in the $\mathrm{CC}$ in patients with chronic atrophic CSC (Fig. 3f).

\section{DISCUSSION}

OCT angiography (OCTA) is a promising, noninvasive imaging technique that enables highresolution and quantitative characterization of the retinal and choroidal vasculature. Recent studies have demonstrated the value of OCTA imaging of the CC in patients with CSC, which are characterized by areas of choroidal hyperand hypoperfusion $[8,12,14,15,17]$. None of those small cohort studies, however, quantitatively assessed the CC OCTA signal in patients with CSC or compared OCTA signals in different subgroups of patients with CSC. Furthermore, none of those investigations sought to identify any association between the OCTA CC signal and subretinal fluid and RPE atrophy, respectively, which are hallmarks of acute and chronic atrophic CSC. The aim of this to-date largest cohort study was therefore to provide a detailed overview of OCTA signal changes at the level of the CC in patients presenting with different stages of CSC and to investigate a potential correlation between SRF and RPE alterations and the OCTA signal.

Our study demonstrates that normal eyes exhibit a relatively consistent CC vascular network as described before $[18,19]$. Patients with CSC, however, exhibit areas of increased and decreased OCTA signals at the level of the CC. This mixed signal pattern in our population is in line with previous OCTA choriocapillaris studies describing areas of reduced and enhanced CC flow in patients with CSC $[8,12,14,15,17]$. Only one study reported a different pattern characterized by a predominantly increased OCTA signal in the CC that corresponded with leakage in fluorescein angiography [16]. This discrepancy may be attributed to different OCTA 

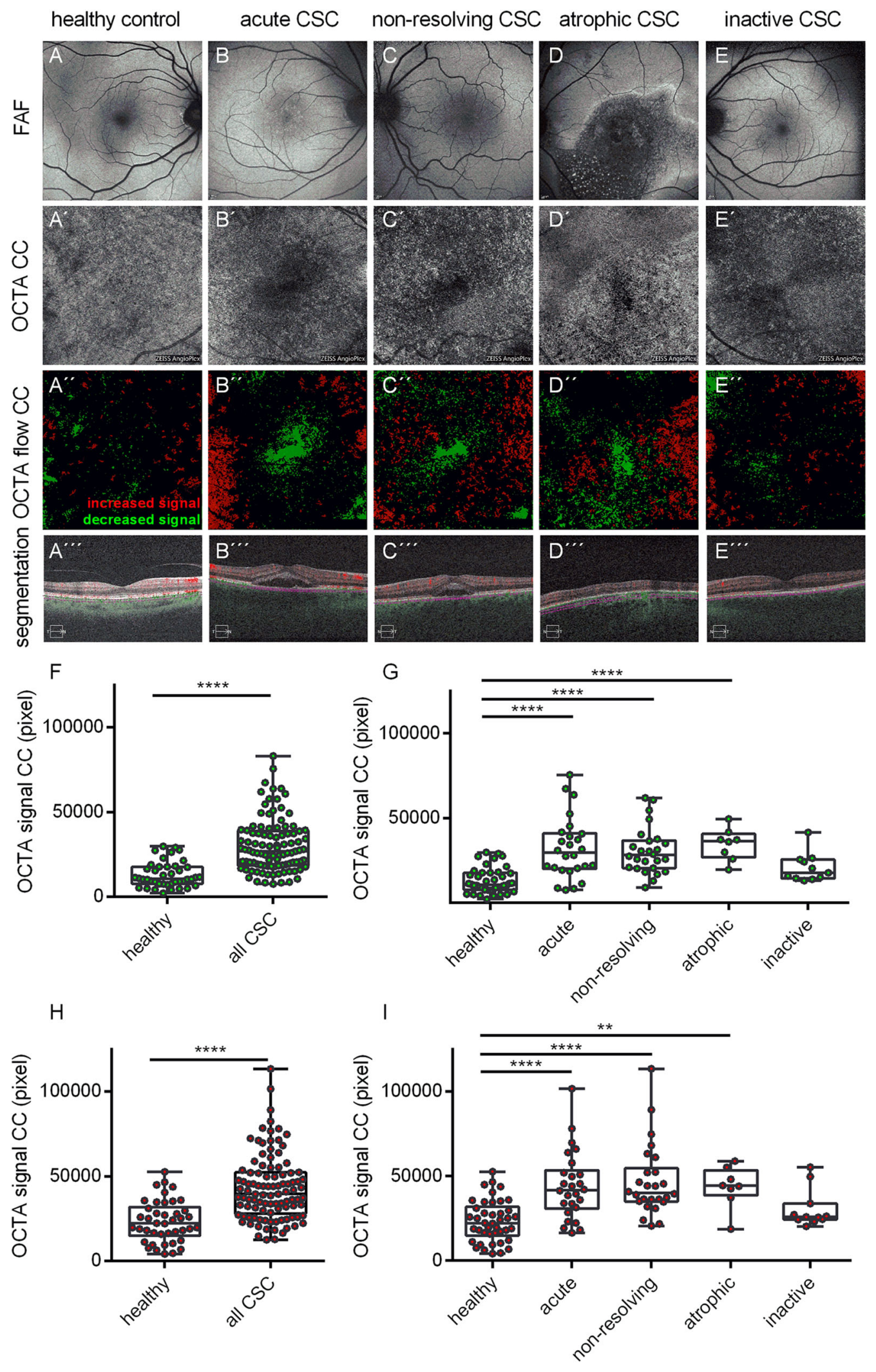
4Fig. 1 Patients with active central serous chorioretinopathy (CSC) demonstrate both increased and decreased OCTA signals at the level of the choriocapillaris (CC) compared with inactive CSC and control patients. Fundus autofluorescence (FAF, top row), OCTA image of the CC and flow pseudocoloration (middle rows) and OCTA segmentation (bottom row) of control patients (a), acute CCS (b), chronic non-resolving CSC (c), chronic atrophic CSC (d), and inactive CSC (e). f-i Increased CC OCTA signals are pseudocolored in red, decreased OCTA signals in green. Box and whisker plots demonstrating the number of pixels of decreased (f, $\mathbf{g})$ and increased OCTA signal (h, i) in all patients and in CSC subgroups. Each dot represents one eye. Mann-Whitney $t$ test $(\mathbf{f}, \mathbf{h})$ and ANOVA/Bonferroni multiple comparison test $(\mathbf{g}, \mathbf{i})$. ${ }^{* *} p<0.01,{ }^{* * * *} p<0.0001$

devices used in the studies or by the presence of secondary choroidal neovascularization, which is characterized by an increased OCTA signal [20] and was not obviously excluded by Chan and colleagues [16].

Our subgroup analysis revealed that all active CSC subgroups with subretinal fluid displayed significantly higher numbers of increased and decreased OCTA signals in the CC compared with age-matched controls. Patients with inactive CSC, in contrast, showed less OCTA alteration, similar to healthy age-matched controls, suggesting that the $\mathrm{CC}$ architecture returns to normal following active CSC. This finding is surprising in light of the residual changes in fundus autofluorescence, fluorescein angiography, and SD-OCT imaging [4], and the functional alterations of the $\mathrm{CC}$ in patients with inactive CSC [21]. Given the small number of patients with inactive CSC in our study, however, we cannot draw any firm conclusions. Further multimodal imaging studies with a greater number of patients are needed to clarify
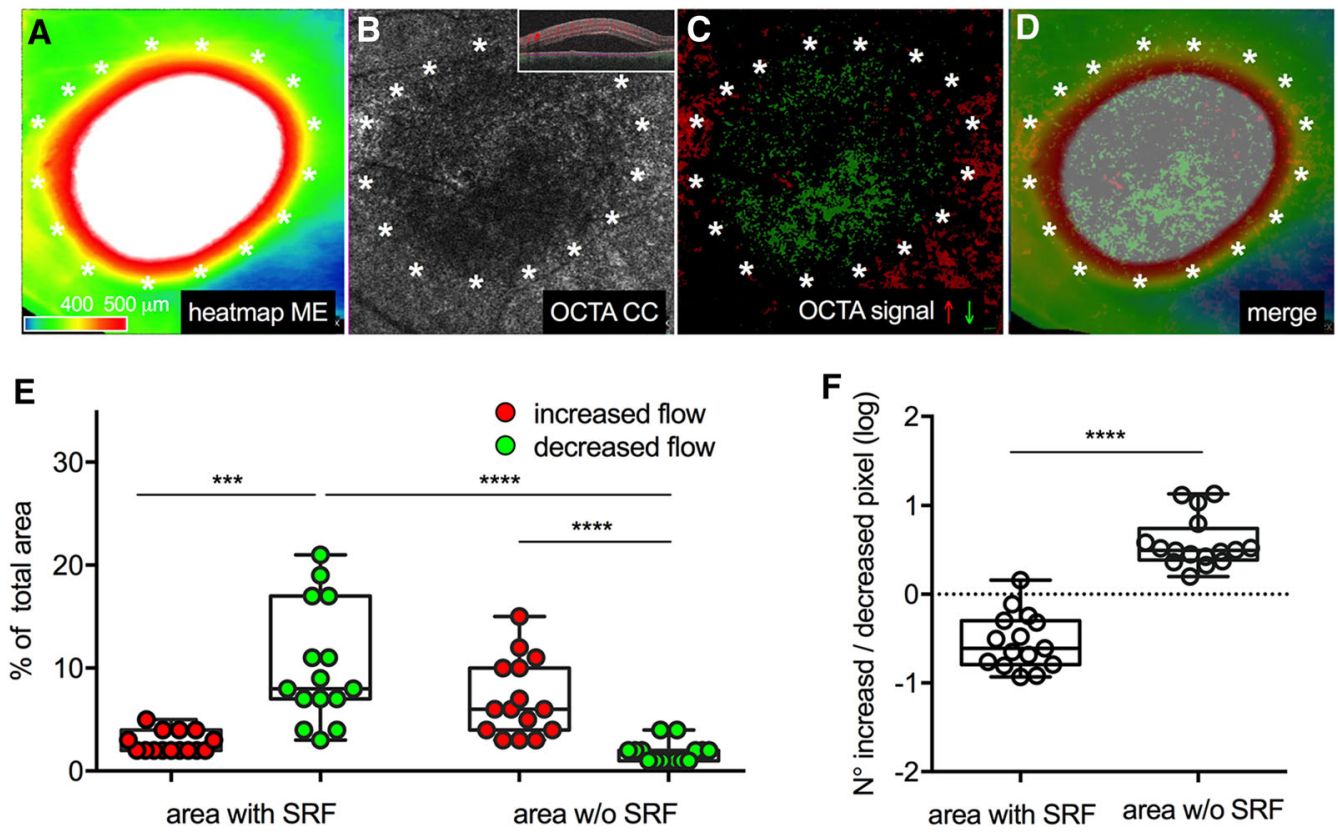

Fig. 2 Patients with acute CSC demonstrate increased OCTA signals outside the area of subretinal fluid (SRF) and decreased OCTA signals in the area with SRF. Macular edema heatmap (a), corresponding OCTA image without (b) and with (c) pseudocoloration demonstrating areas of increased (green) and decreased flow (red), and a merged image (d) of a representative patient with acute CSC. Asterisks surround the area of increased SRF defined as $>450 \mu \mathrm{m}$ macular thickness. e Box and whisker blot demonstrating the relative number of increased (red) and decreased flow (green) in the area with and without SRF. f Ratio of increased to decreased pixels $(\log )$ in areas with and without SRF. Each dot represents one eye $(n=15)$. ANOVA/Dunn post hoc test (e) and Mann-Whitney $t$ test $(\mathbf{f}) .{ }^{* * *} p<0.001,{ }^{* * * *} p<0.0001$ 

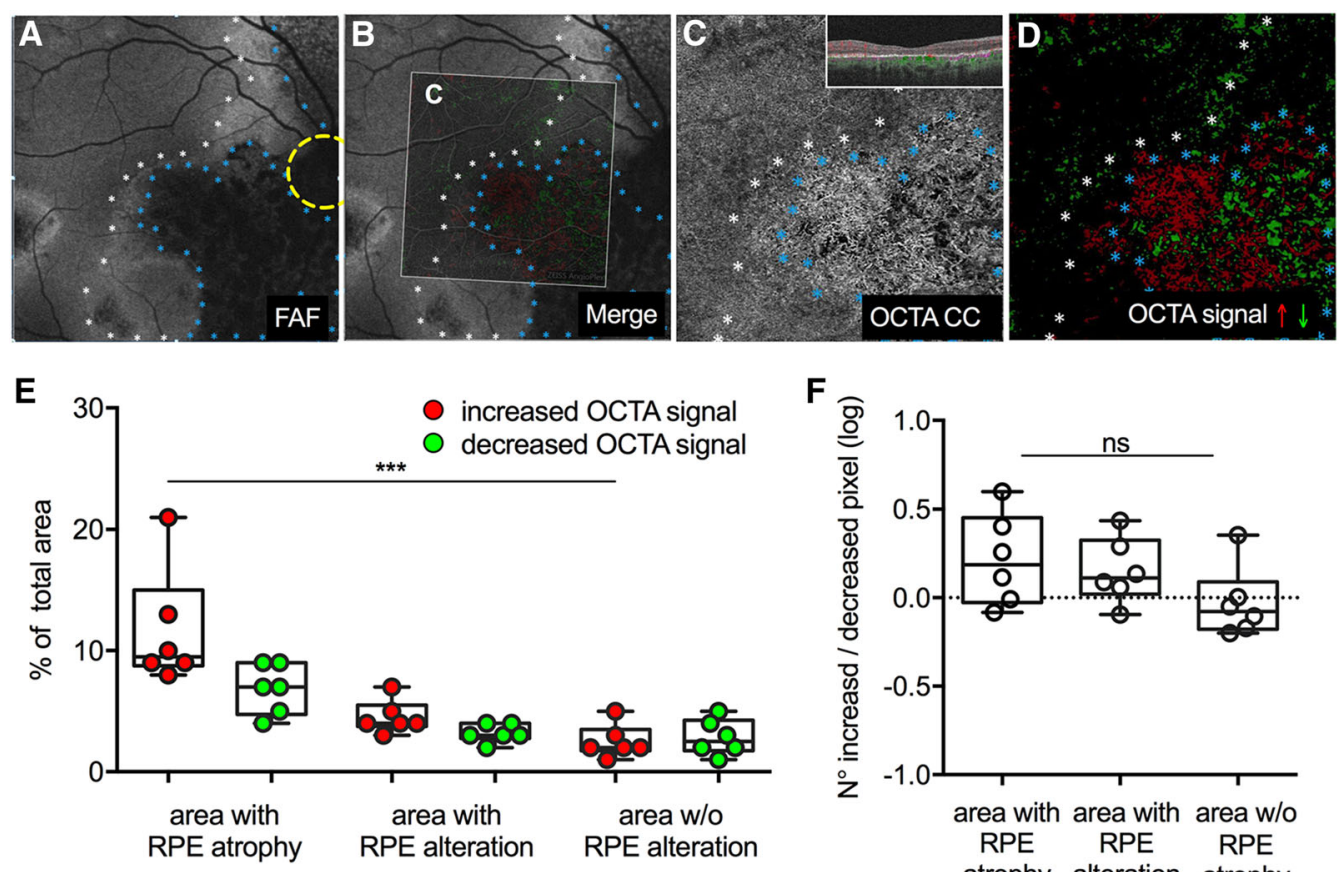

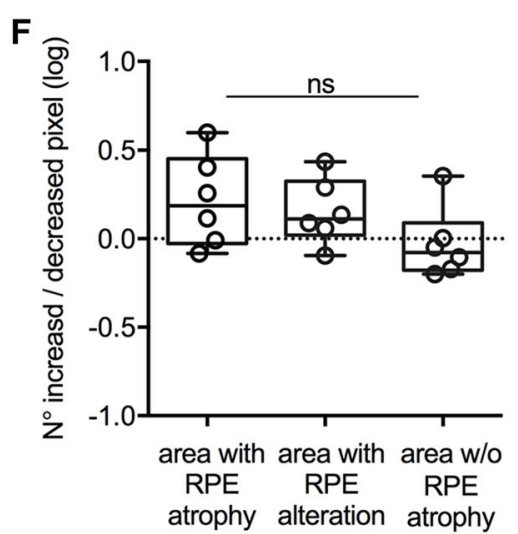

Fig. 3 RPE atrophy co-localizes with areas of increased choriocapillaris (CC) OCTA signal in patients with atrophic CSC, while areas revealing RPE alterations defined by increased fundus autofluorescence exhibit a normal OCTA signal. Fundus autofluorescence (FAF) image (a), FAF OCTA CC merge (b), naive (c), and pseudocolored OCTA image (d) of a representative patient with chronic atrophic CSC. Increased CC OCTA signals are pseudocolored in red, decreased OCTA signals in green. Blue asterisks surround the area of RPE atrophy,

the state of the $\mathrm{CC}$ in patients with inactive CSC.

The results of this study confirm a mixed OCTA signal pattern with areas of increased and decreased OCTA signals in the CC of patients with CSC, in line with previous reports $[8,12,14,15,17]$. To determine any interdependence between OCTA signals and the subretinal fluid that is a hallmark of CSC, we determined the spatial correlation between the OCTA signal and subretinal fluid in patients with acute CSC. Intriguingly, we found a strong spatial correlation between areas of reduced OCTA signals and subretinal fluid, while only a small percentage of increased-flow pixels were detected within the area of SRF.

This finding may be explained by either a reduced blood flow in the $\mathrm{CC}$ or a shadowing white asterisks surround the area with RPE changes. The yellow-dotted line marks the optic nerve head. e Box and whisker plots demonstrating the relative number of increased (red) and decreased flow (green) in the area with RPE atrophy, RPE alteration, and normal RPE. f Ratio of increased to decreased pixels (log) in areas with RPE atrophy, RPE alteration, and normal RPE. Each dot represents one patient $(n=6)$. ANOVA/Dunn post hoc test $(\mathbf{d}, \mathbf{e}) .{ }^{* * *} p<0.001$

artifact. A fact arguing against a purely artificial cause is the non-homogeneous appearance of the OCTA signal in the area of high domeshaped subretinal fluid (as depicted in Fig. 2a-d). In particular, under the assumption that SRF causes shadow artifacts (i.e., decreased OCTA signal), the detection of increased OCTA signal in the area of SRF is unexpected. Furthermore, we did not observe a gradient in the OCTA pattern reflecting the height of the SRF and the dome shape of the SRF bleb. The observed areas of increased or decreased OCTA signals were not spatially associated with the height of SRF, arguing against a purely artificial cause and shadowing artifact. It is conceivable that impaired flow in the CC may hamper fluid absorption, causing subretinal fluid to accumulate during the acute stage of the disease. FAG/ 
ICG- and Doppler flowmetry-based studies demonstrating reduced choroidal blood flow compared with the unaffected eye in CSC patients support this hypothesis $[22,23]$. However, even though our and previous data indicate a hypoperfusion of the CC as a primary disease pathology, we must be cautious in the interpretation of decreased OCTA signal $[12,24]$. Decreased OCTA could be caused by shadowing artifacts related to subretinal fluid, pigment epithelial detachment, elongation, or irregularities of the photoreceptor outer segment-all factors that cannot be ruled out with confidence and need to be addressed in future studies.

Finally, our study assessed the spatial correlation between the OCTA signal in the CC and areas of RPE atrophy in patients with chronic atrophic CSC. We found that patients with chronic atrophic CSC reveal profound CC OCTA signal abnormalities in the area of RPE atrophy, but no significant differences in the adjacent hyperfluorescent area. In geographic atrophy of age-related macular degeneration (AMD), discussions have focused on whether RPE atrophy leads to CC atrophy or vice versa [25-27]. CC OCTA studies of geographic atrophy patients revealed severely decreased CC flow in areas of RPE atrophy [18, 28, 29]. In line with these findings are results of a histologic study of human samples from McLeod et al. that demonstrated a 50\% loss of CC density in regions of complete RPE atrophy and postulated linear CC reduction related to RPE loss in regions of geographic atrophy [25]. However, no complete CC atrophy in the area of RPE atrophy was detected in this study. The assessment of CC atrophy is due to the dynamic range of detectable blood flow by OCTA limited in our study [18]. The predominant increased OCT signal in the area of RPE atrophy in our analysis is not in line with the histologic data by McLeod et al. and previous OCTA geographic atrophy studies. This could be explained by inward displacement of the high-flow deeper choroid layer in the CC slab through thinning and/or atrophy of the CC. Interestingly, we observed a normal CC OCTA signal in the area of increased fundus autofluorescence adjacent to the RPE atrophy, which is characterized by accumulation of lipofuscin fluorophores and discussed as a sign of RPE malfunction leading to RPE atrophy $[30,31]$. The missing OCTA signal changes in this area in our analysis favor the RPE over the CC as the primary site of injury. In contrast to our findings, Pellegrini et al. revealed persisting lobules with reduced flow at the margin of the RPE atrophy in Stargardt patients [29]. The use of the lower resolution $6 \times 6$ volume scan and mean grayscale value-dependent quantification algorithm of each individual image in our study may have failed to detect subtle changes within this area.

Our study's limitations include its retrospective study design, the relatively small number of patients in different subgroups, and the possibility that artifacts were a contributing cause to choriocapillary flow changes. Shadowing artifacts by SRF, PED, and photoreceptor outer segment irregularities could have contributed to the predominately decreased flow signal in acute and non-resolving CSC patients. Furthermore, the predominant increased flow signal in chronic atrophic CSC patients could have been explained by structural changes such as loss of the optical dense RPE and translocation of the high-flow deeper choroid layer in the CC slab. Another limitation of our study is the use of the averaged pixel intensity of each image to determine increased and decreased flow changes within the same image, which may have caused some over- and underestimation of flow changes in unaffected areas. Prospective longitudinal studies with optimized image acquisition techniques less prone to artifact are therefore necessary to further validate our findings.

\section{CONCLUSION}

In summary, the present study reports on the largest cohort, OCTA-based CC assessment to date and is the first to quantify CC flow changes in different subgroups of CSC. Patients with active CSC presented significantly increased and decreased flow pixel intensities in the CC compared to patients with inactive CSC and age-matched healthy controls. Surprisingly, no changes were found within the active CSC 
groups. The decreased flow in the SRF area in acute CCS patients could be evidence of a reduced blood flow in the CC in an active state or due to shadowing artifacts. The lack of CC changes in RPE areas revealing increased autofluorescence adjacent to RPE atrophy argues against primary CC injury. An analysis relying on higher resolution images specifically designed to investigate changes in the margin of the RPE atrophy is warranted to assess this question more accurately.

\section{ACKNOWLEDGEMENTS}

Funding. No funding or sponsorship was received for this study or publication of this article. The article processing charges were funded by the authors.

Authorship. All named authors meet the International Committee of Medical Journal Editors (ICMJE) criteria for authorship for this article, take responsibility for the integrity of the work as a whole, and have given their approval for this version to be published.

Author Contributions. Concept and design: BC, CL; data acquisition: BC, CL, MR, SL, AB, $\mathrm{CE}, \mathrm{BG}, \mathrm{MS}, \mathrm{SR}, \mathrm{AS}$; data analysis/interpretation: $\mathrm{BC}, \mathrm{CL}, \mathrm{MR}, \mathrm{DB}$; drafting manuscript: $\mathrm{BC}, \mathrm{CL}$; critical revision of manuscript: $\mathrm{MR}, \mathrm{SL}, \mathrm{AB}, \mathrm{CE}$, BG, MS, SR, AS, HA; supervision: CL, HA. All authors read and approved the final manuscript.

Disclosures. Bertan Cakir, Michael Reich, Stefan Lang, Anima Bühler, Christoph Ehlken, Bastian Grundel, Milena Stech, Sabine Reichl, Andreas Stahl, Daniel Böhringer, Hansjürgen Agostini, and Clemens Lange have nothing to disclose.

Compliance with Ethics Guidelines. All procedures performed in studies involving human participants were in accordance with the Freiburg University Hospital review board and with the 1964 Helsinki Declaration and its later amendments or comparable ethical standards. Informed consent was obtained from all individual participants included in the study.

Data Availability. All data generated or analyzed during this study are included in this published article/as supplementary information files.

Open Access. This article is distributed under the terms of the Creative Commons Attribution-NonCommercial 4.0 International License (http://creativecommons.org/licenses/ by-nc/4.0/), which permits any noncommercial use, distribution, and reproduction in any medium, provided you give appropriate credit to the original author(s) and the source, provide a link to the Creative Commons license, and indicate if changes were made.

\section{REFERENCES}

1. Wang M, Munch IC, Hasler PW, Prünte C, Larsen M. Central serous chorioretinopathy. Acta Ophthalmol (Copenh). 2008;86:126-45.

2. Kitzmann AS, Pulido JS, Diehl NN, Hodge DO, Burke JP. The incidence of central serous chorioretinopathy in Olmsted county, Minnesota, 1980-2002. Ophthalmology. 2008;115:169-73.

3. Daruich A, Matet A, Behar-Cohen F. Central serous chorioretinopathy. Dev Ophthalmol. 2017;58:27-38.

4. Daruich A, Matet A, Dirani A, Bousquet E, Zhao M, Farman N, et al. Central serous chorioretinopathy: recent findings and new physiopathology hypothesis. Prog Retin Eye Res. 2015;48:82-118.

5. Bousquet E, Beydoun T, Zhao M, Hassan L, Offret O, Behar-Cohen F. Mineralocorticoid receptor antagonism in the treatment of chronic central serous chorioretinopathy: a pilot study. Retina Phila Pa. 2013;33:2096-102.

6. Zhao M, Célérier I, Bousquet E, Jeanny J-C, Jonet L, Savoldelli $\mathrm{M}$, et al. Mineralocorticoid receptor is involved in rat and human ocular chorioretinopathy. J Clin Invest. 2012;122:2672-9.

7. Alten F, Heiduschka P, Clemens CR, Eter N. Exploring choriocapillaris under reticular pseudodrusen using OCT-angiography. Graefes Arch Clin 
Exp Ophthalmol Albrecht Von Graefes Arch Klin Exp Ophthalmol. 2016;254:2165-73.

8. Teussink MM, Breukink MB, van Grinsven MJJP, Hoyng CB, Klevering BJ, Boon CJF, et al. OCT angiography compared to fluorescein and indocyanine green angiography in chronic central serous chorioretinopathy. Invest Ophthalmol Vis Sci. 2015;56:5229-37.

9. Quaranta-El Maftouhi M, El Maftouhi A, Eandi CM. Chronic central serous chorioretinopathy imaged by optical coherence tomographic angiography. Am J Ophthalmol. 2015;160(581-587):e1.

10. Dansingani KK, Balaratnasingam C, Klufas MA, Sarraf D, Freund KB. Optical coherence tomography angiography of shallow irregular pigment epithelial detachments in pachychoroid spectrum disease. Am J Ophthalmol. 2015;160(1243-1254):e2.

11. Bonini Filho MA, de Carlo TE, Ferrara D, Adhi M, Baumal CR, Witkin AJ, et al. Association of choroidal neovascularization and central serous chorioretinopathy with optical coherence tomography angiography. JAMA Ophthalmol. 2015;133:899-906.

12. Costanzo E, Cohen SY, Miere A, Querques G, Capuano V, Semoun O, et al. Optical coherence tomography angiography in central serous chorioretinopathy. J Ophthalmol. 2015;2015:134783.

13. de Carlo TE, Rosenblatt A, Goldstein M, Baumal CR, Loewenstein A, Duker JS. Vascularization of irregular retinal pigment epithelial detachments in chronic central serous chorioretinopathy evaluated with OCT angiography. Ophthalmic Surg Lasers Imaging Retina. 2016;47:128-33.

14. Shinojima A, Kawamura A, Mori R, Fujita K, Yuzawa M. Findings of optical coherence tomographic angiography at the choriocapillaris level in central serous chorioretinopathy. Ophthalmol J Int Ophtalmol Int J Ophthalmol Z Für Augenheilkd. 2016;236:108-13.

15. Feucht N, Maier M, Lohmann CP, Reznicek L. OCT angiography findings in acute central serous chorioretinopathy. Ophthalmic Surg Lasers Imaging Retina. 2016;47:322-7.

16. Chan SY, Wang Q, Wei WB, Jonas JB. Optical coherence tomographic angiography in central serous chorioretinopathy. Retina Phila Pa. 2016;36:2051-8.

17. Cakir B, Reich M, Lang SJ, Bühler A, Stahl A, Böhringer $\mathrm{D}$, et al. Possibilities and limitations of OCTangiography in patients with central serous chorioretinopathy. Klin Monatsbl Augenheilkd. 2017;234:1161-8.
18. Choi W, Mohler KJ, Potsaid B, Lu CD, Liu JJ, Jayaraman V, et al. Choriocapillaris and choroidal microvasculature imaging with ultrahigh speed OCT angiography. PLoS One. 2013;8:e81499.

19. Braaf B, Vienola KV, Sheehy CK, Yang Q, Vermeer $\mathrm{KA}$, Tiruveedhula $\mathrm{P}$, et al. Real-time eye motion correction in phase-resolved OCT angiography with tracking SLO. Biomed Opt Express. 2013;4:51-65.

20. Bousquet E, Bonnin S, Mrejen S, Krivosic V, Tadayoni R, Gaudric A. Optical coherence tomography angiography of flat irregular pigment epithelium detachment in chronic central serous chorioretinopathy. Retina. 2018;38(3):629-638

21. Tittl M, Maar N, Polska E, Weigert G, Stur M, Schmetterer L. Choroidal hemodynamic changes during isometric exercise in patients with inactive central serous chorioretinopathy. Invest Ophthalmol Vis Sci. 2005;46:4717-21.

22. Kitaya N, Nagaoka T, Hikichi T, Sugawara R, Fukui $\mathrm{K}$, Ishiko $\mathrm{S}$, et al. Features of abnormal choroidal circulation in central serous chorioretinopathy. Br J Ophthalmol. 2003;87:709-12.

23. Scheider A, Nasemann JE, Lund OE. Fluorescein and indocyanine green angiographies of central serous choroidopathy by scanning laser ophthalmoscopy. Am J Ophthalmol. 1993;115:50-6.

24. Spaide RF, Fujimoto JG, Waheed NK. Image artifacts in optical coherence tomography angiography. Retina Phila Pa. 2015;35:2163-80.

25. McLeod DS, Grebe R, Bhutto I, Merges C, Baba T, Lutty GA. Relationship between RPE and choriocapillaris in age-related macular degeneration. Invest Ophthalmol Vis Sci. 2009;50:4982-91.

26. Saint-Geniez M, Kurihara T, Sekiyama E, Maldonado $\mathrm{AE}, \mathrm{D}^{\prime}$ Amore PA. An essential role for RPEderived soluble VEGF in the maintenance of the choriocapillaris. Proc Natl Acad Sci USA. 2009;106:18751-6.

27. Schlingemann RO. Role of growth factors and the wound healing response in age-related macular degeneration. Graefes Arch Clin Exp Ophthalmol Albrecht Von Graefes Arch Klin Exp Ophthalmol. 2004;242:91-101.

28. Kvanta A, Casselholm de Salles M, Amrén U, Bartuma H. Optical coherence tomography angiography of the foveal microvasculature in geographic atrophy. Retina Phila Pa. 2017;37:936-42.

29. Pellegrini M, Acquistapace A, Oldani M, Cereda MG, Giani A, Cozzi M, et al. Dark atrophy: an optical coherence tomography angiography study. Ophthalmology. 2016;123:1879-86. 
30. Sparrow JR, Kim SR, Cuervo AM, Bandhyopadhyayand U. A2E, a pigment of RPE lipofuscin, is generated from the precursor, A2PE by a lysosomal enzyme activity. Adv Exp Med Biol. 2008;613:393-8.
31. McLeod DS, Taomoto M, Otsuji T, Green WR, Sunness JS, Lutty GA. Quantifying changes in RPE and choroidal vasculature in eyes with age-related macular degeneration. Invest Ophthalmol Vis Sci. 2002;43:1986-93. 